\title{
Comparison of Online Distribution Channels of the Thailand Community Enterprise Products
}

\author{
Rataphol Sangkhasuk, Kanlaya Naklungka, Wiriyaporn Ekphon, Worapun Surasawadee \\ Phranakhon Rajabhat University, Bangkok, Thailand
}

\begin{abstract}
This research aims to study an online distribution of community enterprise products, to design the management system for an e-commerce distribution channel, and to compare the efficiency of distribution channels through a developed e-commerce system and ready web. A personal interview survey method was used to collect data on the production, marketing, and online selling issues. In total 150 community enterprise entrepreneurs in five product categories (food, appliance/home decoration, beverage, garment/ornament and non-food herb) and 50 consumers were equally selected by judgment sampling method. The e-commerce channel that was developed is our website and social network website that was selected to compare the efficiency of distribution channels is Facebook. The research found that only $14.67 \%$ of community enterprise entrepreneurs sell products through online channel. The product which was sold through the developed website and maximized gross profit is food $(35.53 \%$ of total revenue). The others, are beverage, non-food herb, appliance/home decoration, and garment/ornament, earn gross profit $31.50 \%, 22.39 \%, 9.78 \%$ and $0.80 \%$ of total revenue, respectively. The result of distribution channels comparison between our website and webpages on Facebook found that Facebook has the ability to reach customers 2,811 times more than the website $64.75 \%$, which has only 991 times. Regarding to sales, it is found that revenue from Facebook is higher than the website in all product categories.
\end{abstract}

Keywords: community enterprise products, e-commerce, online channel

\section{Introduction}

The Thai Government has recently initiated the project called community enterprise from the gathering of the locals in order to manage their well-being with the idea of self-reliance. Most activities of the project are planned to help the local people manage their own resources, such as local wisdoms inherited from their ancestors, local herbs, community cultures, etc. However, the challenge of the community enterprise is that, apart from its productivity and service, it lacks the channel for product distribution. As a result, most community enterprise groups have to rely on commission merchants. Should the groups be able to distribute

Rataphol Sangkhasuk, master of Engineering (Industrial Engineering), Phranakhon Rajabhat University, Bangkok, Thailand.

Kanlaya Naklungka, master of Science (Agricultural Economics), Faculty of Management Science, Phranakhon Rajabhat University, Bangkok, Thailand.

Wiriyaporn Ekphon, master of Agro-industry (Agro-industrial technology), University Business Incubator (UBI), Phranakhon Rajabhat University, Bangkok, Thailand.

Worapun Surasawadee, master of Education (Art Education), Faculty of Humanities and Social Science, Phranakhon Rajabhat University, Bangkok, Thailand.

Correspondence concerning this article should be addressed to Rataphol Sangkhasuk, Faculty of Industrial Technology, Phranakhon Rajabhat University, 9 Changwattana Road, Bangkhen Bangkok 10220, Thailand. 
their own goods, they would earn as much incomes as they expect to gain.

The Government also encourages the community where the local could rely on themselves to be stronger through information technology mechanism. For instance, there is the support from the government with the project called "Digital OTOP" which sponsors the local potential producers to begin their marketing online. The workshops and counseling in using social media are provided. This includes starting their website for e-commerce and other social media. The project is aimed at the local wisdom preservation groups with the potential to reach international markets so as to promote local culture, identity, and wisdom taken to be the rudimentary design of the products. Phranakhon Rajabhat University as the university for the locals plays an active role in helping the community stronger, which is led by the Institute of Research and Development, Pranakhon Rajabhat University. The University has set up the cluster of Thai OTOP distributors in order to strengthen the community. Also, the University is made aware of the problem of community enterprise concerning the channel of product distribution and increase of the sale value. In 2014, Thanakij Kokthong, Suparak Suwanwat, and Nirat Sudsang have conducted the research called "The initiation of digital photographing art to promote the sale via Facebook." This study aims at evaluating the satisfaction of entrepreneurs and customers and comparing the satisfaction between a new way of product initiation and the traditional one (Tanakit, Suparug, \& Nirat, 2014). In 2004, Khomkrit Yasamut and Namphon Nanthapreechakul have presented the management system for e-commerce website of tamarind product factory called Sarach Marketing Co. Ltd. This project is to find the way to develop the information and news presentation through website and internet service (Nanthapreechakul, 2004). In 2004, Charupan Chulahong and Sudarat Saengkaew have studied the application of e-commerce system to Thai Balm Co. Ltd. in order to improve the business competitiveness to meet up with the demand of today's market, ranging from public relations, marketing, information exchange between partner and back-end office management (Chulahong \& Saengkaew, 2004). The findings reveal the importance of distributing channel development to deal with the expansion of community enterprise and it is crucial for the government to issue any policy to support this project so as to prepare itself for ASEAN Economic Community in the future and to strengthen the community to be able to rely on themselves.

\section{Methodology}

The research was divided into two parts: the analysis of current situation of selling products via e-commerce and the comparison of channels for selling products via e-commerce.

(1) The survey of current situation of selling community enterprise's products is conducted in order to derive the information from questionnaires and then plan the strategies of selling products via e-commerce. The surveyor randomly selected 200 persons and divided them into two groups: 150 entrepreneurs which are sub-divided into five OTOP groups with 30 persons each and 50 customers. The questionnaires are analyzed with the use of SPSS program.

(2) Two channels for selling products via e-commerce are compared: the development of their own websites and the selling via Facebook. A total of 70 products were selected to launch in those channels to see which online channel is the most effective by the following steps.

\section{The Development of Their Own Website}

The process of developing their own website for selling products via e-commerce is as follows:

The design of website's name or domain. The research team chose the website's name from OTP Trend 
Assistant and then registered the domain and web address under the name of www.otopta.com, which is easy to read and not too long. The meaning of which is understandably linked to products. Also, the logo for the website is designed to make it recognizable as showed in Figure 1.

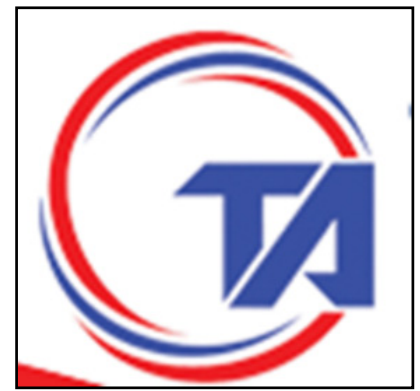

Figure 1. Logo for website (www.otopta.com).

Designing the website. There are two mains steps which require the web-creator to follow in order to design the website for e-commerce.

(1) We can divide the designing of the e-commerce into two sections: front store and back store as shown below in Figure 2.

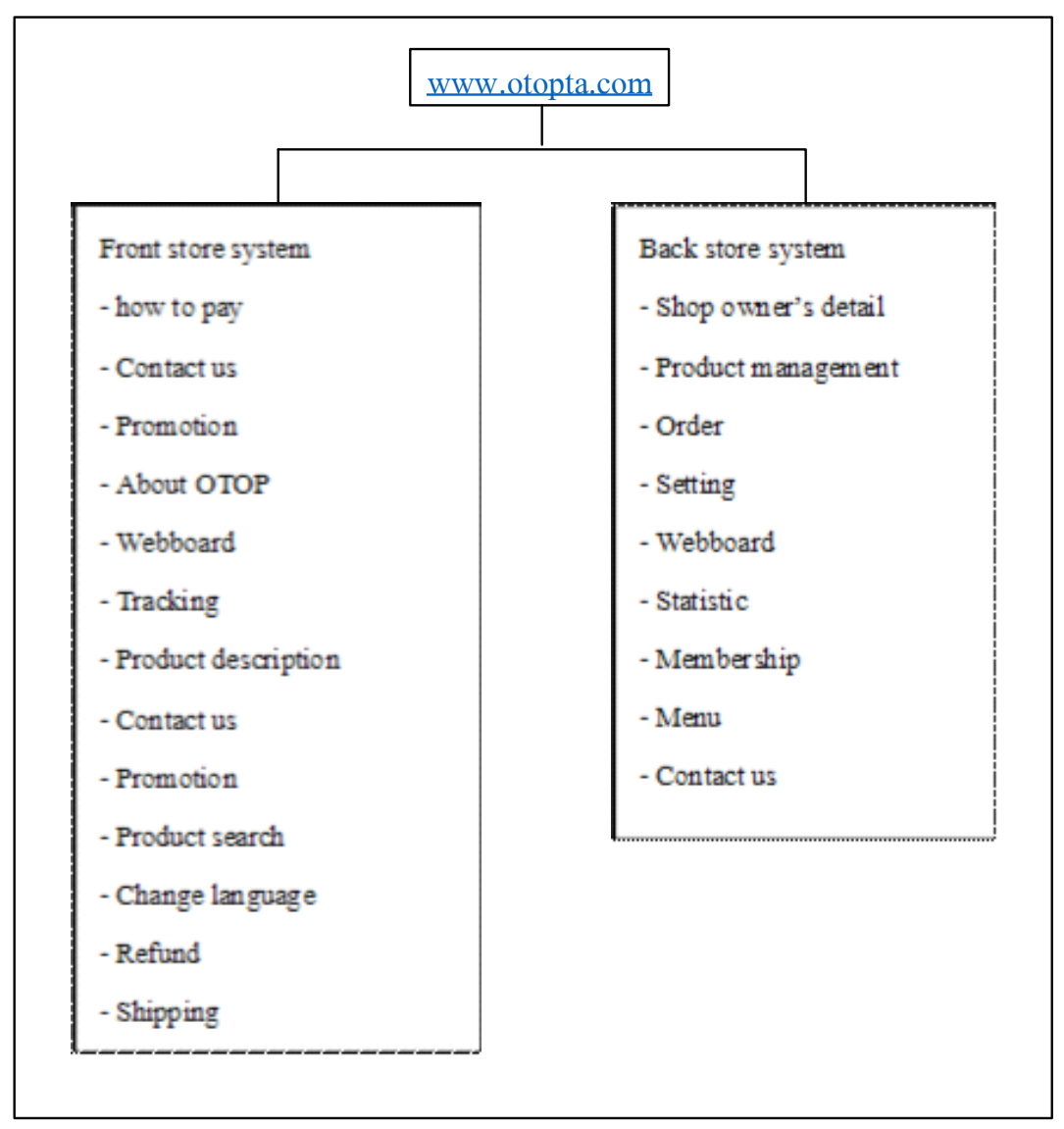

Figure 2. The designing of the menu for e-commerce website.

As we can see from Figure 2, the designing of the e-commerce website called www.otopta.com is divided into two main sections. 
- Front store comprises the following menus: How to pay, Contact us, Promotion, About OTOP, Web board, Tracking, Product description, and Product search.

- Back store comprises the following menus: Shop owner's detail, Product management, Order, Setting, Web board, Statistic, Membership, Menu, and Contact us.

(2) The designing of user interface

This step is divided into three sections as follows:

- The website heading or banner consists of name and logo of the website, searching box, change language icon (which can be switched between Thai and English), search icon, how to order icon and upper menu tab which has the following shot cut keyboard: home, list of product by types, and contact us as shown in Figure 3 .

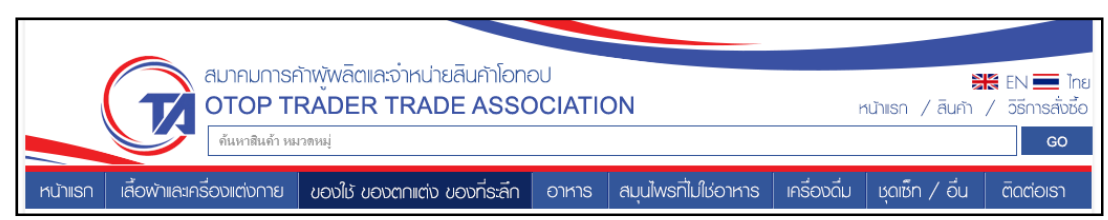

Figure 3. The designing of the website's heading.

- The middle part of the website consists of promotion, new products, icon for selecting products by type, "about us" menu on the left hand side which also serves as a shot cut. This section consists of category, about us, about OTOP, web board, how to pay, contact us, and tracking as shown in Figure 4.

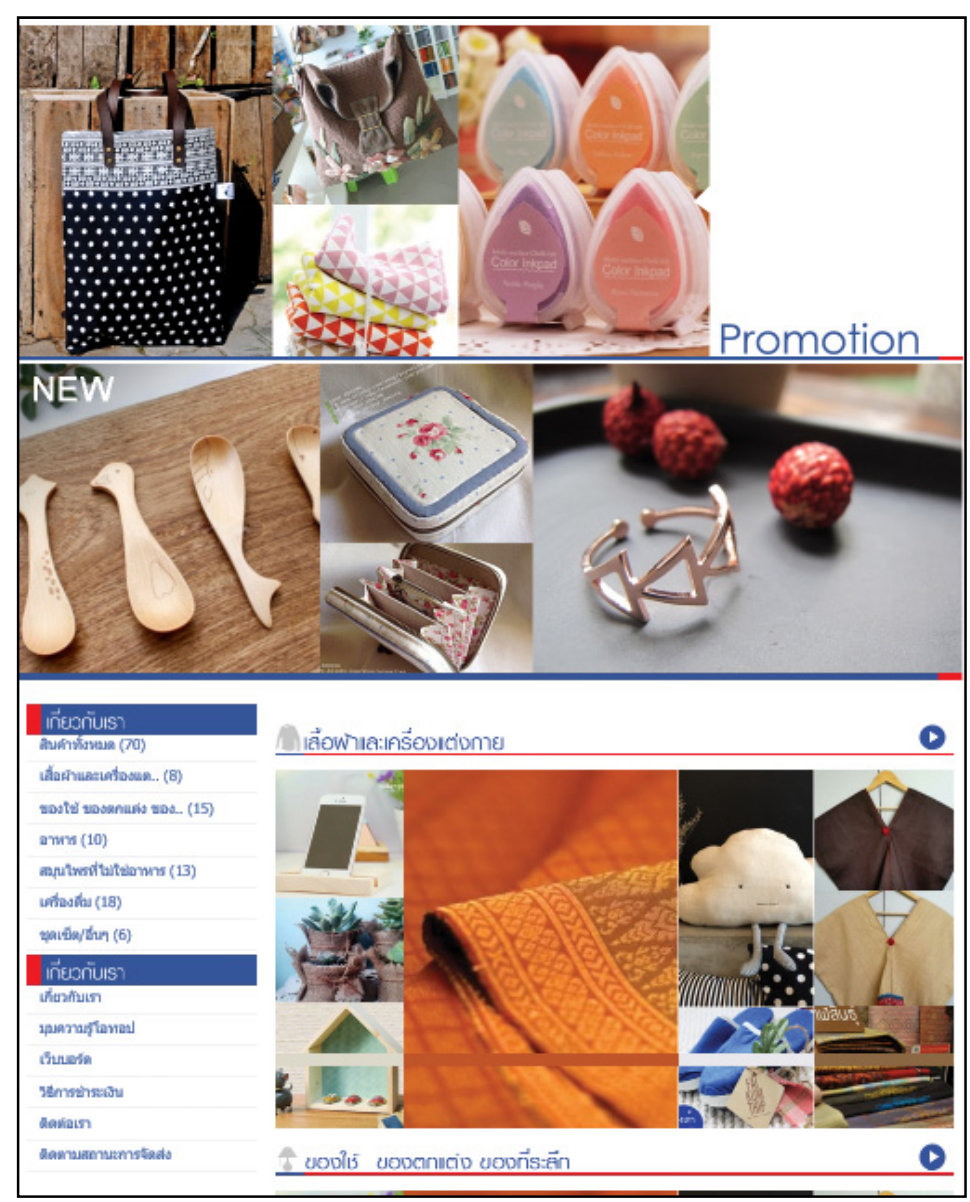

Figure 4. The designing of the middle part of website. 
- The bottom part of the website consists of customer service, category, and seller's address. The designing of this part is shown below in Figure 5.

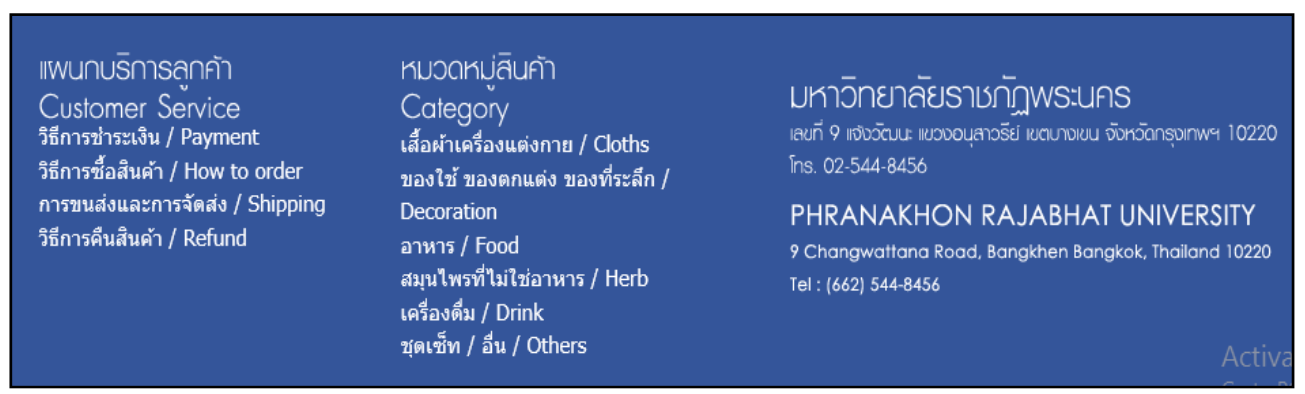

Figure 5. The designing of the bottom part of the website.

\section{The Method of Designing}

The e-commerce system via social network is to create a platform in the popular social media because there are various features to be employed to advertise the product, such as sharing on Facebook's account and easily forwarding to others. Therefore, the research team chose Facebook as an alternative to compare with other online channels. In the Facebook's design for selling product, it is called OTOPTA, which has the webpage as shown in Figure 6.

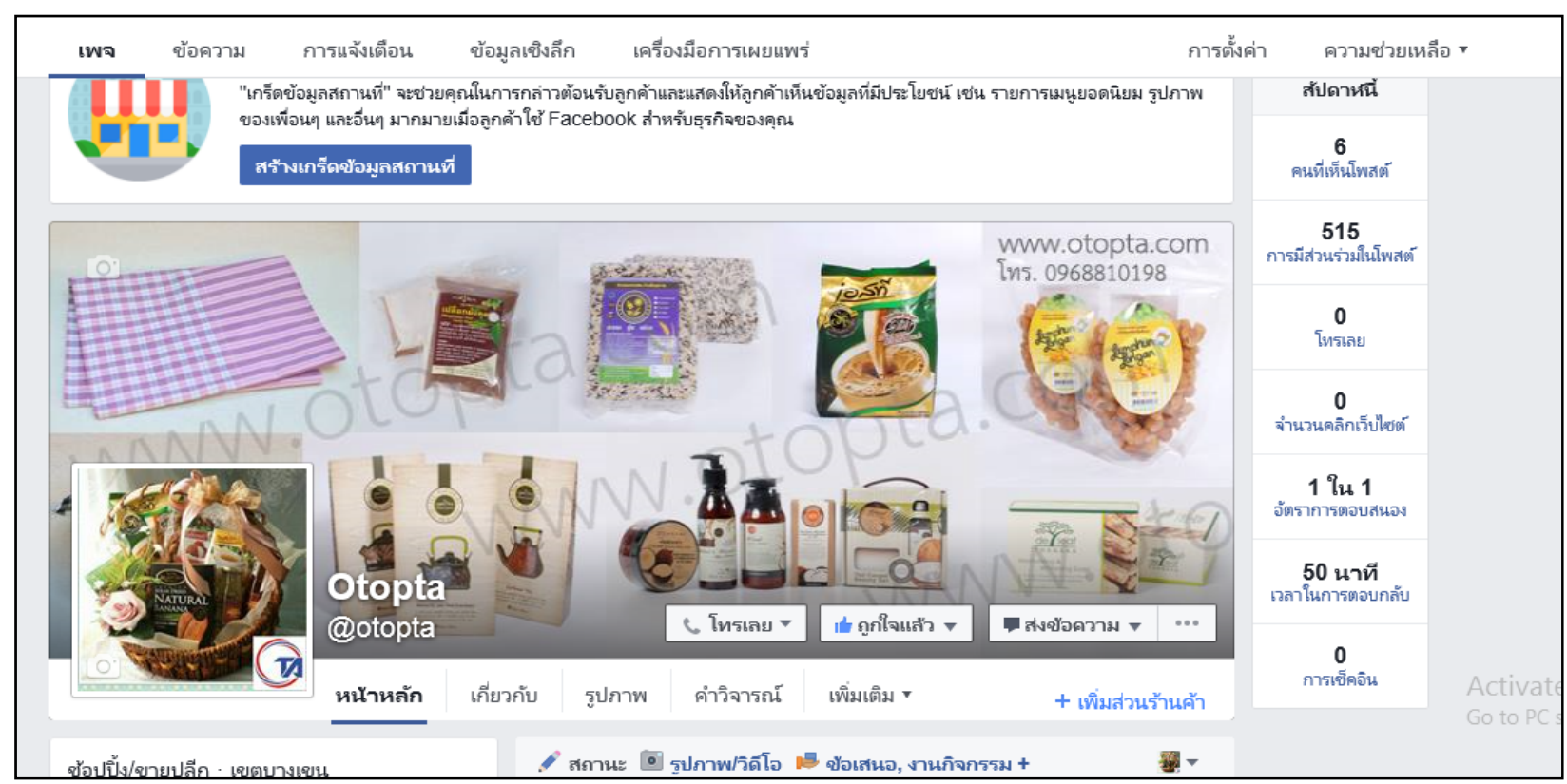

Figure 6. Facebook webpage of OTOPTA.

\section{The Analysis of Distribution Channels}

In developing the online distribution system, the research team analyzed the category of community enterprise's products to see whether they are suitable for selling online and analyzed to find the advisable strategies for each category of community enterprise's products. Also, the study compared two online selling channels: website and Facebook. It employed the comparing methods as follows:

- The number of customer who has access to these channels indicates the chance of customer to visit the website. 
- The amount of product sold via these channels indicates the customer's quality that can assess to these channels.

\section{Conclusion and Discussion}

\section{Conclusion}

The study found that after the interview session with 150 entrepreneurs, there were only 22 community enterprise entrepreneurs who used online selling system, which accounts for 14.67 per cent. Food section made the most profit, accounting for 35.53 per cent of total sale value. The second most profiting section is beverage, accounting for 31.5 per cent of total sale value. Herb (not food) is the third profiting section which accounts for 22.39 per cent. Decorated objects and furniture is the fourth with 9.78 per cent of total sale value. Cloth and ornament is the last profiting section with only 0.8 per cent. In terms of comparison of the distribution channels, it is found that Facebook is considered the most accessible channel for online sale, with 2,811 times of visit throughout the period in question; while, the website developed has only 991 times of visit. The difference between the two channels accounts for 1,820 times of visit, or 64.75 percent.

\section{Discussion}

The findings reveal that Facebook is better channel than website for distributing products. It is largely because Facebook is the most convenient channel for customer to assess the products with its features such as sharing, asking information, and ordering. As for the person who controls the page, it is also easy for him/her to maintain and update the Facebook page and make it accessible and quick in response to the customer's demand. This findings resonate with the result from the study (Sriwichailampan, 2013) who conducted the research on the development of online system and found that the customers are likely to get more convenient and quick service after ordering a product and it is easy to advertise itself to the wider customers. This includes the back store system that helps the seller look at the list of product or change any information for customer without delay. The findings are also similar to those from the report of (Thai e-Commerce Association, 2015) in that the e-commerce system is interestingly helpful channel for distributing product. It can sell goods 24 hours a day. It can reach people who live in other parts of the world, and its information can be updated all the time. It is time-saving and economical in advertising and can replace human, store or booth in real life sale, as well as help increase sale volume. Technology helps make products more interesting, open door for other business deal. There is a wide range of products and services to choose from, without having to waste time to commute to the actual store. It can also be noticeable when the mistake occurs and quickly solve them. E-commerce has after sale service too.

\section{References}

Chulahong, C., \& Saengkaew, S. (2004). The development of electronic commerce: Case studies Partnership Thailand Palm. Naresuan University, Phitsanulok.

Sriwichailampan, T. (2013). Teaching academic community economic development. Chiang Mai, Chiang Mai University.

Tanakit, K., Suparug, S., \& Nirat, S. (2014). The creation of digital photography art for the promotion of fashion goods on facebook. Art and Architecture Journal, Naresuan University, 5(1), 26-40.

Thai e-Commerce Association. (2015). Registration of SME and enterprise networking communities manual. Retrieved from http://www.thaiwbi.com/topic/E-Ecommerce/

Yasamut, K., \& Nantapeechakun, N. (2004). Management system web commercial electronic processing plant tamarind sarach. Marketing Company Limited., Naresuan university, Pitsanulok. 